\title{
A refined position catalogue of the Swift XRT afterglows ${ }^{\star}$
}

\author{
A. Moretti ${ }^{1}$, M. Perri ${ }^{2}$, M. Capalbi ${ }^{2}$, L. Angelini ${ }^{3,4}$, J. E. Hill ${ }^{4,5}$, S. Campana ${ }^{1}$, D. N. Burrows ${ }^{6}$, J. P. Osborne ${ }^{7}$, \\ G. Tagliaferri ${ }^{1}$, G. Cusumano ${ }^{8}$, P. Giommi ${ }^{2}$, P. Romano ${ }^{1}$, T. Mineo ${ }^{8}$, J. Kennea ${ }^{6}$, D. Morris ${ }^{6}$, J. Nousek ${ }^{6}$, \\ C. Pagani ${ }^{1,6}$, J. Racusin ${ }^{6}$, A. F. Abbey ${ }^{7}$, A. P. Beardmore ${ }^{7}$, O. Godet ${ }^{7}$, M. R. Goad ${ }^{7}$, K. L. Page ${ }^{7}$, \\ A. A. Wells ${ }^{7}$, and G. Chincarini ${ }^{1,9}$
}

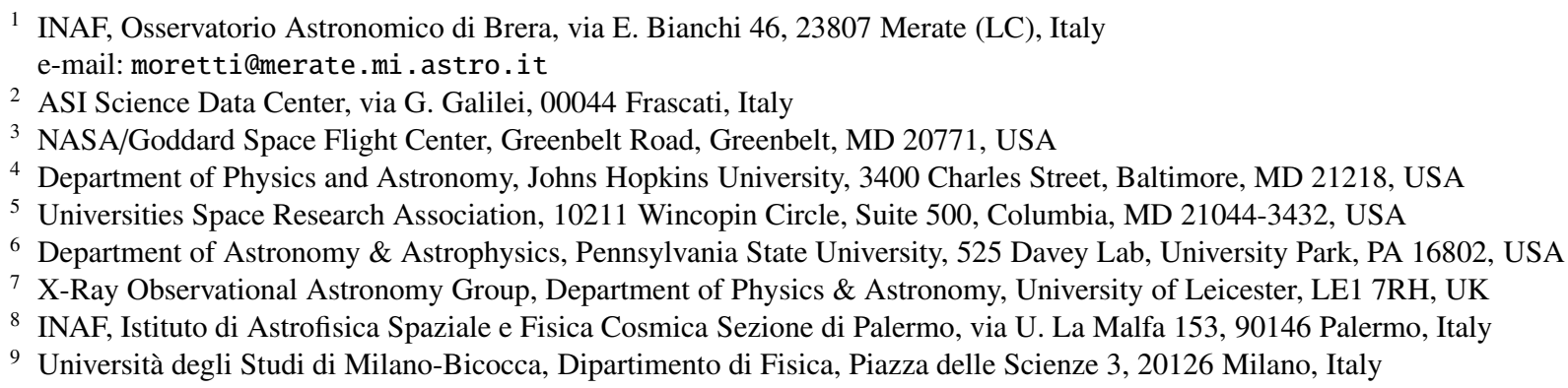

Received 18 November 2005 / Accepted 19 January 2006

\section{ABSTRACT}

We present a catalogue of refined positions of 68 gamma ray burst (GRB) afterglows observed by the Swift X-ray Telescope (XRT) from the launch up to 2005 Oct. 16. This is a result of the refinement of the XRT boresight calibration. We tested this correction by means of a systematic study of a large sample of X-ray sources observed by XRT with well established optical counterparts. We found that we can reduce the systematic error radius of the measurements by a factor of two, from $6.5^{\prime \prime}$ to $3.2^{\prime \prime}$ ( $90 \%$ of confidence). We corrected all the positions of the afterglows observed by XRT in the first 11 months of the Swift mission. This is particularly important for the 37 X-ray afterglows without optical counterpart. Optical follow-up of dark GRBs, in fact, will be more efficient with the use of the more accurate XRT positions.

Key words. Keywords should be given

\section{Introduction}

The Swift satellite (Geherels et al. 2004) detects and localises gamma ray bursts (GRBs) and provides autonomous rapid response observations and long term monitoring of their afterglow emission. The scientific payload consists of three instruments: the Burst Alert Telescope (BAT, Barthelmy et al. 2005), the X-ray Telescope (XRT, Burrows et al. 2005) and the UV/Optical Telescope (UVOT, Roming et al. 2005). Swift observations provide prompt $\gamma$-ray positions with an accuracy of few arcminutes, X-ray positions with an accuracy of few arcseconds and UV/Optical positions with accuracy of less than $1^{\prime \prime}$. From the satellite launch (2004 Nov. 20) to 2005 Oct. 16, XRT observed 64 afterglows of GRBs detected by BAT and 5 afterglows of GRBs detected by other instruments (INTEGRAL and HETE-2).

For the same GRB sample only 31 optical counterparts were found either by UVOT or by ground-based telescopes.

* Table 1 is available in electronic form at the CDS via anonymous ftp to cdsarc.u-strasbg.fr $(130.79 .128 .5)$ or via http://cdsweb.u-strasbg.fr/cgi-bin/qcat?J/A+A/448/L9 and at http://www.edpsciences.org
In most of the remaining cases stringent upper limits were set by deep optical observations. Classical explanations for burst optical darkness are, dust extinction, high redshift or intrinsic faintness. The study of the host galaxies through ultra-deep follow-up observations, is one of the most important ingredients for the determination of GRB progenitors. One of the key factors in the follow-up is the accuracy of the GRB position. Before Swift, the study of the host galaxy was possible only in the presence of an optical afterglow detection (with the exception for the rare cases observed by XMM-Newton, Chandra or by radio telescopes), because of the limited position accuracy of the X-ray and $\gamma$-ray telescopes. This limitation could have produced some bias in the statistical study of the population of the host galaxies. For example, if we assume that the dust extinction plays an important role in the optical obscuration of the afterglow emission, the optically selected sample of host galaxies would have been biased against dust-rich galaxies. Now, by means of the Swift prompt automatic observations and the high quality of the XRT optics, we can obtain X-ray afterglow positions so accurate that the identification of the host galaxies is possible even when only the X-ray afterglow 
position is available. One of the goals of the XRT is to provide afterglow X-ray positions with the unprecedented accuracy of $5^{\prime \prime}$. From a comparison of the XRT positions with the optical transients positions we find that, at present, $90 \%$ of the XRT positions are within a $6.5^{\prime \prime}$ radius error circle, slightly worse than the pre-flight expectations. While the statistical uncertainties in the XRT position determination are well known, we find that the larger fraction of the error has a systematic origin. In the present work we show that we can correct for this systematic error, improving the typical accuracy, and, using the proposed correction, we recalculated $68 \mathrm{X}$-ray afterglow positions.

\section{The XRT position accuracy}

For each GRB, XRT usually produces two position measurements: the on-flight rapid position and the refined on-ground position. The way the satellite works is that when the XRT observations starts promptly after the BAT trigger (100-200 s) and the X-ray flux is bright enough $(\sim 10$ counts per second corresponding to $\sim 5 \times 10^{-10} \mathrm{erg} \mathrm{s}^{-1} \mathrm{~cm}^{-2}$ ), the first position measurement is calculated on-board by the flight software (Hill et al. 2004) and automatically distributed by the GRB Coordinate Network (GCN), typically within a few seconds of the spacecraft slewing and settling on the GRB. The XRT was able to calculate and distribute a rapid position, within 350 seconds from the burst trigger, in 19 cases. All the measured XRT positions are then refined on the ground using all the telemetered photon counting (PC) data from the first segment of the observation and sent again in a new GCN circular (see Hill et al. 2005 for a detailed description of the XRT automatic procedure). The uncertainties in the position measurements are determined by the statistical uncertainty and by the precision of the satellite pointing.

When the background rate is negligible, the statistical error in the position determination depends on the instrumental point spread function (PSF) and on the counts of the source according to the simple formula $U_{\text {stat }} \propto R_{90} / \sqrt{\text { counts, }}$, where $U_{\text {stat }}$ is $90 \%$ accuracy error circle radius and $R_{90}$ is the radius which contains $90 \%$ of the fluence. The XRT statistical positional accuracy has been extensively tested on-ground and verified in flight during the calibration phase with some ad hoc observations (Hill et al. 2005; Moretti et al. 2005). We empirically found that the statistical error at the $90 \%$ level of XRT position measurement is given by the formula $U_{\text {stat }}=R \times$ counts $^{-0.48}$ (Hill et al. 2004), with the parameter $R=23^{\prime \prime}$, in very good agreement with the expectations. It means that for a source with more than 144 counts, the statistical error is less than $2^{\prime \prime}$. To determine the afterglow position, the flight software uses a very short exposure image $(0.1$ or $2.5 \mathrm{~s})$ : this means that the typical source counts to determine a centroid with are $<50$ and therefore such positions are highly affected by statistical uncertainties. In telemetered PC mode data there are usually more than 150 counts and the statistical uncertainties in the on-ground refined position measurements are less than $2^{\prime \prime}$. We note that the expected background counts in a region containing $80 \%$ of the PSF in a typical exposure time of $20 \mathrm{ks}$, in the full energy band $(0.2-10 \mathrm{keV})$, are $\sim 2$ and this fully justifies the previous assumption that the background is negligible.

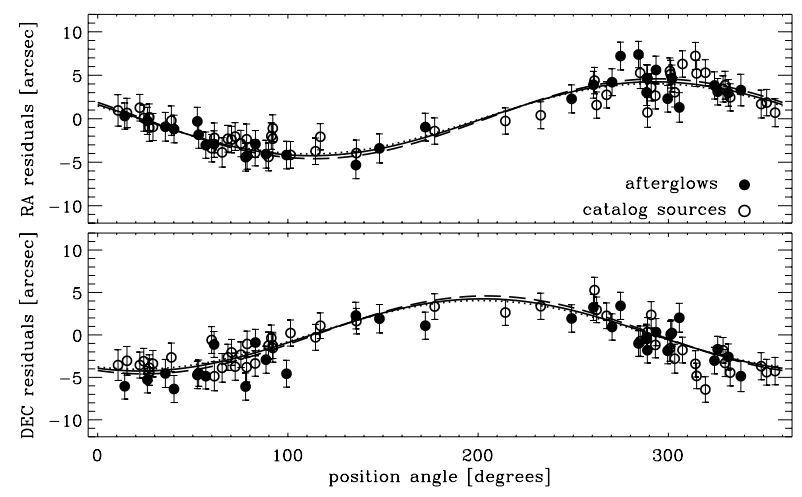

Fig. 1. Differences in coordinates (RA in the upper panel, Dec in the lower) between the XRT and optical positions (X-optical). We used both catalogued sources and GRB observations with well known optical counterpart. The plotted errors are the combination of the statistical errors and the aspect solution uncertainty. The solid line is the fit to the entire sample, the dashed line is the fit relative to the catalogued sources only and the dotted line is the fit to the afterglows: the best fit parameters are perfectly consistent. RA residuals have been corrected by the factor $\cos (\mathrm{Dec})$, and represent the true separation in the sky.

In addition to the statistical error, the XRT position uncertainty is also determined by the uncertainty in the satellite aspect solution. The nominal value of this uncertainty is $3^{\prime \prime}$ as reported in the calibration file swxposerr20010101v002.fits (CALDB version 20050916).

In order to study the systematic errors, we collected all the XRT observations of point-like sources present in the public archive (http://heasarc.gsfc. nasa.gov/cgi-bin/W3Browse/swift.pl) from April to August 2005 with a catalogued optical position in the SIMBAD archive (http://simbad.u-strasbg.fr/Simbad), excluding the few with proper motion. Moreover, we added the 15 calibration observations of Mkn 876, RX J0720.4-3125 and RXS J1708-4009 taken between February 2005 and March 2005. We then considered all the observations of X-ray afterglows with a clearly varying optical counterpart published in the GCN in the period from December 2004 to September 2005. In order to select a very homogeneous sample and to minimize the statistical error, we selected the observations with the source at less than $3^{\prime}$ from the center of the field of view and with more than 150 source counts. It resulted in a sample of 80 observations ( 31 afterglows and 49 catalogued sources). All the XRT data were reduced using the xrtpipeline task of the current release of the HEADAS software (version 1.6), with all the default options and the current release of the calibration files (CALDB version 20050916); then we calculated the XRT positions by means of the xrtcentroid task . As shown in Fig. 1, we found a clear relationship between the spacecraft position angle (PA, also called roll angle) of the observations and the residuals in RA and Dec of the X-ray positions in respect to the optical positions (note that RA residuals have been corrected by the factor cos (Dec), and represent the true separation in the sky). The roll angle of an observation is the angle from sky coordinates to spacecraft coordinates and it is available in the header keyword PA_PNT of the event files. 
We found that this relation is well fitted by a trigonometric function. A similar relation was already found for the MECS and LECS telescopes on board of the BeppoSAX satellite (Perri \& Capalbi 2002). This effect is due to a small calibration error between the XRT boresight and the satellite star tracker boresight, which causes a displacement of the detector system coordinate. The projection of the this displacement in sky coordinates gives a dependence of the coordinates residuals with the roll angle. Therefore, we expect that we can fit together the two relations with the following functions

$$
\begin{aligned}
& \Delta(\mathrm{RA})=M \sin (\mathrm{PA}+\phi) \\
& \Delta(\mathrm{DEC})=M \cos (\mathrm{PA}+\phi)
\end{aligned}
$$

where $M$ is the amplitude of the misalignment and the phase $\phi$ is its direction. The best fit of the data is given by $M=$ $4.2^{\prime \prime} \pm 0.4^{\prime \prime}$ and $\phi=157.6^{\circ} \pm 6.5^{\circ}$ (90\% confidence errors), $\chi^{2}=0.8$ for 158 degrees of freedom. It corresponds to a shift of the nominal detector center of $1.8( \pm 0.2)$ pixels ( 1 pixel is $\left.2.36^{\prime \prime}\right)$. A refinement of the boresight calibration will be included in the standard calibration files from Dec. 2005 CALDB distribution. Because, as explained below, we will use this fit to correct all the afterglow positions, we checked whether the two subsamples (the catalogued sources and the afterglows) yield consistent results. As shown in Fig. 1, the fit of the two different sub-samples, as expected, give perfectly consistent results.

\section{Correction of the systematic error}

The parametrisation of the relationship between the coordinate residuals and the roll angle of the observations (Eqs. (1), (2)) allows us to correct the source position derived from XRT observations with the following:

$$
\begin{aligned}
& \mathrm{RA}_{\text {new }}=\mathrm{RA}_{\text {old }}-\Delta(\mathrm{RA}) / \cos (\mathrm{Dec}) \\
& \mathrm{Dec}_{\text {new }}=\mathrm{Dec}_{\text {old }}-\Delta(\mathrm{Dec}) .
\end{aligned}
$$

First, we tested the goodness of the correction on the 43 sources in the sample with more then 1000 counts (statistical error $<1^{\prime \prime}$, Fig. 2). For all these 43 elements we calculated the distance between the optical position and the refined X-ray positions and we compared it with the uncorrected positions. Because for this particular source sub-sample the statistical uncertainty is negligible, this gives us the measurement of the systematic error.

In Fig. 3 we compare the distribution of the distances before and after the correction. From the integral distribution of the distances we find that $90 \%$ of the X-ray sources after the correction were within a distance of 3.2" (while the uncorrected value was $6.5^{\prime \prime}$ ). This is an improvement by a factor of $\sim 4$ in terms of error circle area. The mean of the distribution changes from $4.3^{\prime \prime}$ to $1.7^{\prime \prime}$ after the correction. We note that, as expected, the corrected residual distributions are well consistent with a Gaussian distribution. The Gaussian fit to the RA residual distribution yields $-0.03 \pm 0.18,1.2 \pm 0.2$ for the mean and standard deviation, respectively $\left(\chi^{2}=0.72\right)$. The Gaussian fit to the DEC residual distribution yields $0.13 \pm 0.20,1.3 \pm 0.2$ for the mean and standard deviation, respectively $\left(\chi^{2}=0.60\right)$.

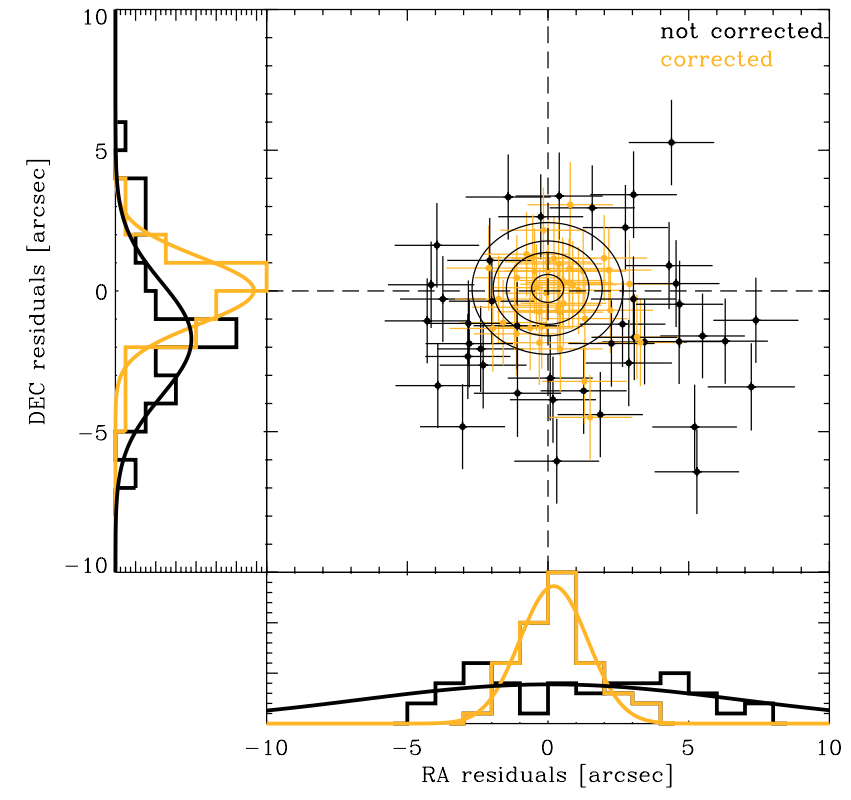

Fig. 2. The residuals of the 43 sources with more than 1000 counts before (black) and after the correction (grey). The contour of the two dimensional Gaussian best fit function are over-plotted in correspondence of $10 \%, 50 \%, 90 \%$ of the maximum value. The external panels show the distribution of the coordinate residuals before and after the correction together with their best Gaussian fit.

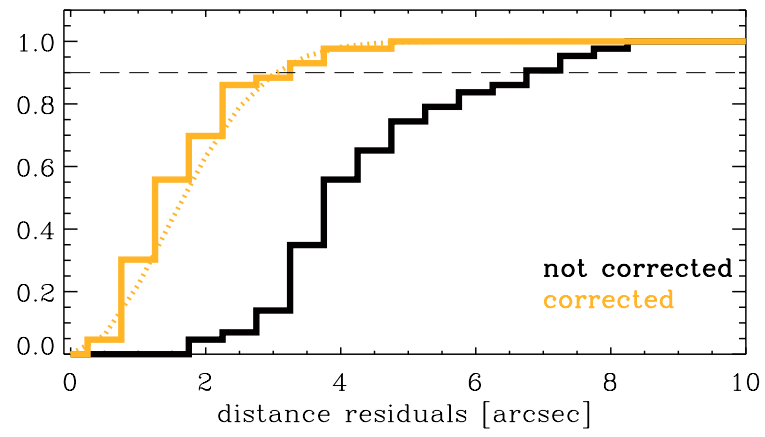

Fig. 3. The integral distribution of the distances of the X-ray sources, with more than 1000 counts, from the optical counterpart before (black lines) and after the correction (grey lines). The dotted line is the the integral of the best Gaussian fit to the distribution of the coordinate residuals (see Fig. 2).

We then applied the boresight correction to the whole sample of 68 afterglows observed by XRT in PC mode from the launch to 2005 Oct. 16. We retrieved all the first segments of the 68 PC observations from the Swift archive. We calculated the XRT positions by means of the xrtcentroid task. This task calculates the source centroids by recursively evaluating the barycentre in boxes reduced by $80 \%$ each time from the initial box size, which is an input parameter. If the statistical significance of the source is very low a too large detection box could affect the position determination. Possible sources of error are a background fluctuations or faint serendipitous sources present in the centroid box. In order to properly take into account for these effects we used the following procedure. First, we calculated the centroid with a fixed input error box size $\left(30^{\prime \prime}\right)$; 
then we refined this position using different box sizes as function of the source counts, ranging from $30^{\prime \prime}$ (for bright sources) to $12^{\prime \prime}$ (for faint sources): for each source we used an initial box size such that the ratio between source and background counts, within the box, is always greater than 20, taking into account both the background rate and the PSF profile (Moretti et al. 2005). Finally we applied the boresight corrections (Eqs. (3), (4)) to the so calculated positions.

The refined positions are reported in Table 1; the quoted errors $(90 \%$ confidence) are the quadratic sum of the statistical error with the new 3.2" systematic error. Moreover in the final error budget we added a term which depends on the source counts and which takes into account the uncertainty caused by the choice of the size of the xrtcentroid error box. We stress, however, that this term is negligible in most of the cases. The mean error of the sample is $3.7^{\prime \prime}$, and $90 \%$ of the afterglows have $90 \%$ confidence uncertainty less than $5^{\prime \prime}$. We stress that among the 68 new positions, 37 are of dark GRBs. We excluded GRB 050117 from our analysis because it does not have any useful PC mode data.

As an example of our results, in Fig. 4 we illustrate the case of GRB 050904 (Cummings et al. 2005), at redshift $z=6.3$ (Kawai et al. 2005). In this case the prompt observations from the ground allowed the detection of the IR transient (D'Avanzo et al. 2005). As shown in Fig. 4 the IR source position is right at the border of the original XRT error circle ( 6 " radius, Mineo et al. 2005), while it is perfectly contained by the refined one (3.2" $)$. The measured colours of this afterglow do not show any hint of dust extinction (Tagliaferri et al. 2005). Had GRB 050904 been highly extincted, it probably would have been undetectable by optical or IR observations. The search of the host galaxy would have been very hard because, although very close, the correct position at the border of the uncorrected XRT error circle.

\section{Conclusion}

We refined the boresight calibration of the XRT, resulting in a significant improvement of the position accuracy. The comparison with optical counterparts shows that on average we reduced the distance between XRT positions and optical positions by a factor of 2 . This was possible by reducing the systematic error from $6.5^{\prime \prime}$ to $3.2^{\prime \prime}$ (90\% confidence). By means of this correction we recalculated the position of 68 afterglows observed by XRT (the complete sample up to 2005 Oct. 16). With one example, we showed how a deep follow-up study of the host galaxies of optically dark GRB can be performed with greater efficiency employing this correction. We note that after Oct. 16 2005 the XRT afterglow positions provided by the XRT team in the GCNs are calculated with the new boresight calibration. Therefore, this work provides the complete catalogue of the refined XRT afterglow positions of the GRB preceding that date. For the subsequent observations of GRB afterglows, we stress that a refined boresight calibration will be implemented in the standard calibration products from the Dec. 2005 CALDB distribution.

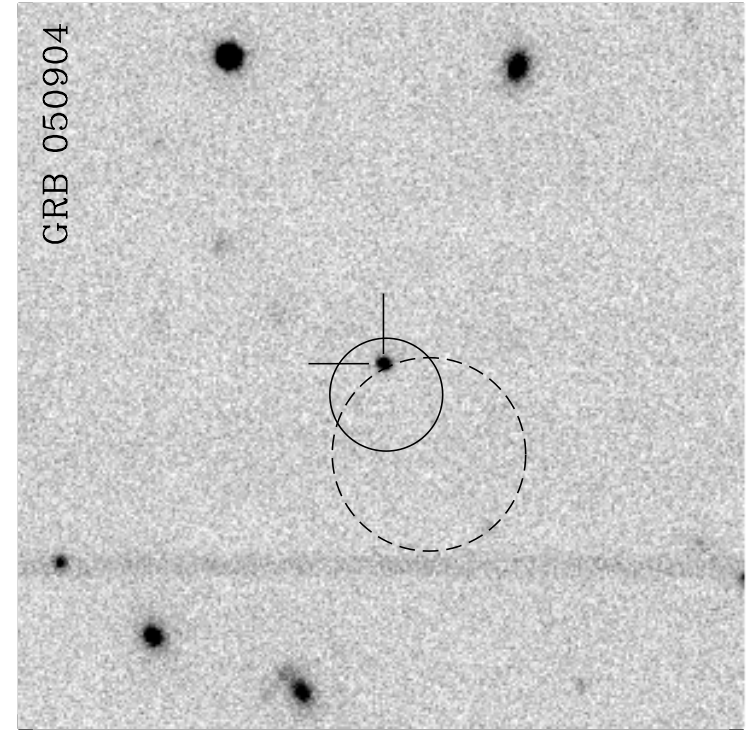

Fig. 4. A VLT+ISAAC J filter image of GRB 050904. The lines show the position of the optical afterglow. The dashed circle is the original error circle, while the smaller and solid line circle is the refined one. The optical transient is at $1.6^{\prime \prime}$ from the refined XRT position and at $6.2^{\prime \prime}$ from the original position quoted in Mineo et al. (2005). As it is clear from the distribution of the residuals (Fig. 3), this represents an extreme case: only $15 \%$ of the positions are expected to be at more than 6 " from the optical position before the correction. The image is taken from Tagliaferri et al. (2005).

Acknowledgements. This work is supported at OAB-INAF by ASI grant I/R/039/04, at Penn State by NASA contract NAS5-00136 and at the University of Leicester by PPARC of grant PPA/Z/S/2003/00507. This research has made use of the SIMBAD database, operated at CDS, Strasbourg, France.

\section{References}

Barthelmy, S. D., Barbier, L. M., Cummings, J. L., et al. 2005, Space Sci. Rev., 120, 143

Burrows, D. N., Hill, J. E., Nousek, J. A., et al. 2005, Space Sci. Rev., 120,165

Cummings, J., Angelini, L., Barthelmy, S., et al. 2005, GCN, 3910

D’Avanzo, P., Antonelli, L. A., Covino, S., et al. 2005, GCN, 3921

Gehrels, N., Chincarini, G., Giommi, P., et al. 2004, ApJ, 611, 1005

Kawai, N., Yamada, T., Kosugi, G., Hattori, T., \& Aoki, K. 2005, GCN, 3937

Hill, J. E., Burrows, D. N., Nousek, J. A., et al. 2004, Proc. SPIE, 5165,217

Hill, J. E., Angelini, L., Morris, D. C., et al. 2005, Proc. SPIE, 5898, 313

Mineo, T., Mangano, V., La Parola, V., et al. 2005, GCN, 3920

Moretti, A., Campana, S., Mineo, T., et al. 2005, Proc. SPIE, 5898, 348

Perri, M., \& Capalbi, M. 2002, A\&A, 396, 753

Roming, P. W. A., Kennedy, T. E., Mason, K. O., et al. 2005, Space Sci. Rev., 120, 95

Tagliaferri, G., Antonelli, L. A., Chincarini, G., et al. 2005, A\&A, 443, L1 
A. Moretti et al.: Refined X-ray positions, Online Material p 1

\section{Online Material}


A. Moretti et al.: Refined X-ray positions, Online Material p 2

Table 1. Refined positions and $90 \%$ statistical and systematic position error radii for the whole sample of 68 GRB afterglows observed by the Swift XRT.

\begin{tabular}{|c|c|c|c|c|c|c|c|}
\hline 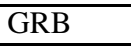 & RA(J2000) & 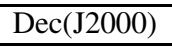 & unc. $(90 \%)\left[{ }^{\prime \prime}\right]$ & GRB & $\begin{array}{l}\mathrm{RA}(\mathrm{J} 2000) \\
\end{array}$ & $\bar{D} \operatorname{Dec}(\mathrm{J} 2000)$ & unc. $(90 \%)\left[{ }^{\prime \prime}\right]$ \\
\hline 041223 & 064047.43 & -370425.2 & 3.4 & 050713B & 203115.51 & +605644.6 & 3.3 \\
\hline 050124 & 125130.33 & +130242.8 & 3.5 & 050714 & 025422.93 & +690646.3 & 5.1 \\
\hline 050126 & 183227.13 & +422214.7 & 3.5 & 050714B & 111847.66 & -153248.9 & 3.3 \\
\hline 050128 & 143817.78 & -344552.6 & 3.2 & 050716 & 223420.77 & +384103.0 & 3.3 \\
\hline 050215B & 113747.70 & +404747.1 & 4.5 & 050717 & 141724.60 & -503200.0 & 3.3 \\
\hline 050219 & 110539.03 & -404100.8 & 4.1 & 050721 & 165344.62 & -282252.1 & 3.3 \\
\hline 050219B & 052515.87 & -574529.9 & 3.4 & 050724 & 162444.64 & -273225.3 & 3.4 \\
\hline 050223 & 180533.08 & -622820.5 & 5.4 & 050726 & 132011.95 & -320350.6 & 3.3 \\
\hline 050306 & 184914.48 & -090910.0 & 4.9 & 050730 & 140817.22 & -034618.8 & 3.2 \\
\hline 050315 & 202554.13 & -423559.8 & 3.2 & 050801 & 133635.37 & -215542.1 & 3.4 \\
\hline 050318 & 031850.77 & -462344.8 & 3.3 & 050802 & 143705.84 & +274710.8 & 3.2 \\
\hline 050319 & 101647.80 & +433254.9 & 3.2 & 050803 & 232237.90 & +054708.8 & 3.2 \\
\hline 050326 & 002749.18 & -712214.6 & 3.6 & 050813 & 160757.07 & +111454.2 & 6.5 \\
\hline 050401 & 163128.85 & +02 1114.4 & 3.3 & 050814 & 173645.43 & +462022.3 & 3.3 \\
\hline 050406 & 021752.39 & -501114.9 & 3.8 & 050815 & 193422.94 & +090850.8 & 3.5 \\
\hline 050408 & 120217.35 & +105109.6 & 3.3 & 050819 & 235501.45 & +245135.3 & 3.7 \\
\hline 050410 & 055912.74 & +793609.8 & 4.0 & 050820 & 222938.16 & +193335.1 & 3.2 \\
\hline 050412 & 120425.19 & -011200.4 & 4.2 & 050820B & 090225.48 & -723843.3 & 4.1 \\
\hline 050416 & 123354.63 & +210327.3 & 3.3 & 050822 & 032427.09 & -460159.6 & 3.3 \\
\hline 050421 & 202903.18 & +733918.2 & 3.5 & 050824 & 004856.23 & +223631.2 & 3.4 \\
\hline 050422 & 213754.92 & +554645.3 & 3.7 & 050826 & 055101.49 & -023838.6 & 3.4 \\
\hline 050502B & 093010.01 & +165947.1 & 3.3 & 050827 & 041709.58 & +18 1200.2 & 3.4 \\
\hline 050504 & 132401.19 & +404215.7 & 5.3 & 050904 & 005450.82 & +140508.2 & 3.2 \\
\hline 050505 & 092703.19 & +301622.7 & 3.2 & 050908 & 012150.65 & -125719.0 & 3.4 \\
\hline 050509 & 204219.86 & +540416.3 & 4.6 & 050915 & 052644.81 & -280100.3 & 3.4 \\
\hline 050509B & 123613.56 & +285901.7 & 7.6 & 050915B & 143626.26 & -672432.1 & 3.4 \\
\hline 050520 & 125005.77 & +302703.7 & 4.7 & 050916 & 090356.98 & -512546.6 & 3.3 \\
\hline 050522 & 132034.63 & +244720.4 & 9.2 & 050922B & 002313.23 & -053617.2 & 3.2 \\
\hline 050525 & 183232.63 & +262021.5 & 3.4 & 050922C & 210933.12 & -084529.6 & 3.3 \\
\hline 050603 & 023956.82 & -251055.2 & 3.5 & 051001 & 232348.77 & -313120.9 & 3.5 \\
\hline 050607 & 200042.78 & +090830.5 & 3.3 & 051006 & 072314.02 & +09 3019.5 & 3.4 \\
\hline 050701 & 150901.67 & -592453.7 & 3.7 & 051008 & 133129.50 & +4205 55.7 & 3.2 \\
\hline 050712 & 051048.00 & +645448.0 & 3.3 & 051016 & 081116.65 & -181755.1 & 3.5 \\
\hline 050713 & 212209.78 & +770428.9 & 3.3 & 051016B & 084827.70 & +133918.8 & 3.3 \\
\hline
\end{tabular}

